\title{
Dilated Coronary Sinus: An Indicator of Persistent Left Superior Vena
}

Cava

Otero $\mathrm{D}^{1}$, Stoddard $\mathrm{M}^{1}$, Ikram $\mathrm{S}^{1^{*}}$

${ }^{1}$ Cardiovascular Medicine Department, University of Louisville, Louisville, KY, USA

Corresponding Author: Sohail Ikram MD, FACC

Address: 201 Abraham Flexner Way, Suite 6oo, Louisville, KY 40202, USA; Tel: +1 502-852-1845; E-mail: s.ikram@louisville.edu

Received date: 24 April 2020; Accepted date: 02 May 2020; Published date: 12 May 2020

Citation: Otero D, Stoddard M, Ikram S. Dilated Coronary Sinus: An Indicator of Persistent Left Superior Vena Cava. Asp Biomed Clin Case Rep. 2020 May 12;3(2):116-117.

Copyright (C) 2020 Otero D, Stoddard M, Ikram S. This is an open-access article distributed under the Creative Commons Attribution License, which permits unrestricted use, distribution, and reproduction in any medium provided the original work is properly cited.

\section{Abstract}

An 88-year-old male presented for a routine transthoracic echocardiogram. Dilated coronary sinus was noted, raising the suspicion for persistent left superior venous cava (PLSVC). An agitated saline study from the left upper extremity demonstrated a flow through the coronary sinus into the right ventricle in a parasternal long-axis view. A venogram from the right internal jugular vein showed the PLSVC drained into a much dilated coronary sinus (CS) that connected to the right atrium. The right superior vena cava was absent. PLSVC along with absent right superior vena cava is rare and the inadvertent CS cannulation may result in vessel perforation.

\section{Keywords}

Congenital Anomaly, Venous Anomaly, Transthoracic Echocardiogram

\section{Case}

An 88-year-old male with aortic stenosis status post bioprosthetic valve replacement presented for routine transthoracic echocardiography (TTE) to evaluate shortness of breath. Severe mitral regurgitation with a left ventricular ejection fraction of $55 \%$ and severe pulmonary hypertension (PH) with pulmonary systolic pressure of $60 \mathrm{mmHg}$, were identified. The aortic prosthetic valve was well seated and with normal gradients. A dilated coronary sinus was noted during examination raising the suspicion of a persistent left superior venous cava (PLSVC), (Fig-1, Video-1) so an agitated saline study was carried out. An intravenous catheter was placed in the left antecubital vein and agitated saline was injected, demonstrating flow through the coronary sinus into the right ventricle, in parasternal long- axis view (Fig-2, Video-2). A right heart catheterization was arranged to evaluate his pulmonary hypertension, revealing Group II PH. During the procedure, a Swan-Ganz catheter was noted to cross the mid-parasternal line. A venogram from the right internal jugular vein showed the PLSVC from merger of the right and left brachiocephalic vein. The PLSVC drained into a much dilated coronary sinus (CS) that connected to the right atrium (Fig-3, Video3). The right superior vena cava (SVC) was absent.

PLSVC is a rare congenital anomaly reported in $0.1 \%$ of the general population and, in the vast majority of cases; right SVC is present [1]. PLSVC along with absent right SVC is extremely rare. It results from failure of the vessel to involute during embryologic development and is found incidentally during procedures requiring SVC access. Inadvertent 
Citation: Otero D, Stoddard M, Ikram S. Dilated Coronary Sinus: An Indicator of Persistent Left Superior Vena Cava. Asp Biomed Clin Case Rep. 2020 May 12;3(2):116-117.

\section{Image Article}

CS cannulation may result in vessel perforation or arrhythmia. Our patient was referred for MitraClip procedure so recognizing his anatomic venous variant was utmost to plan ahead of the procedure.

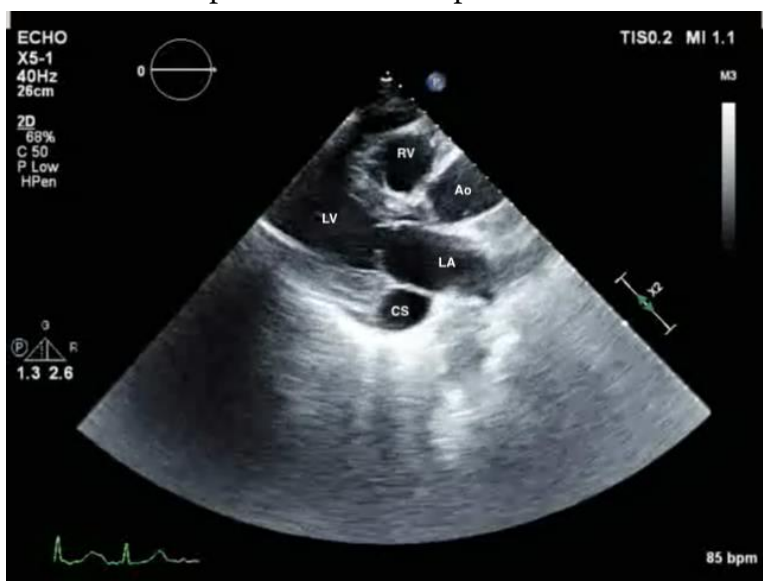

Fig-1:

Two-dimensional transthoracic echocardiogram with a parasternal long view showing dilated coronary sinus, raising the suspicion for persistent left superior vena cava.

LV: Left Ventricle; LA: Left Artium; CS: Coronary Sinus; $R V$ : Right Ventricle; AO: Aorta

The video related to this can be found online at: Video-1

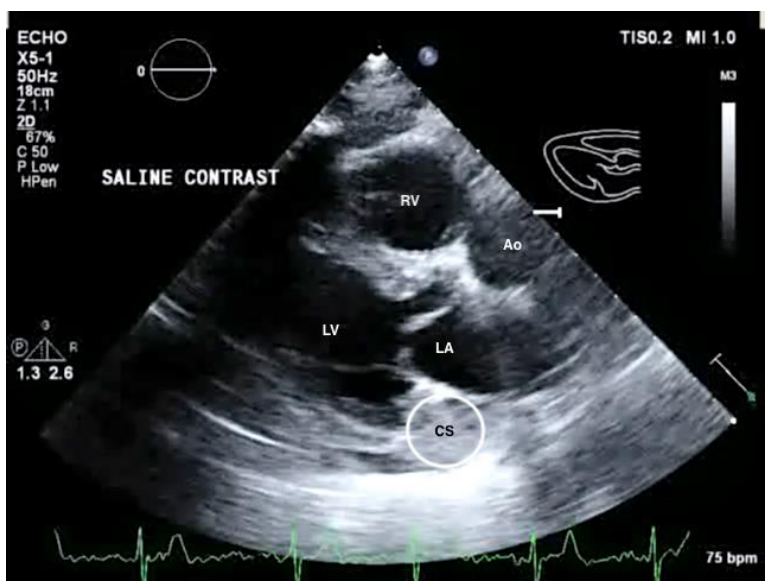

Fig-2:

Two-dimensional transthoracic echocardiogram with a parasternal long view showing an injection of saline contrast through an IV catheter in the left upper extremity demonstrates saline contrast entering sinus prior to the right ventricle, confirming persistent left superior vena cava.

LV: Left Ventricle; LA: Left Artium; CS: Coronary Sinus; $R V$ : Right Ventricle; AO: Aorta

The video related to this can be found online at: Video-2

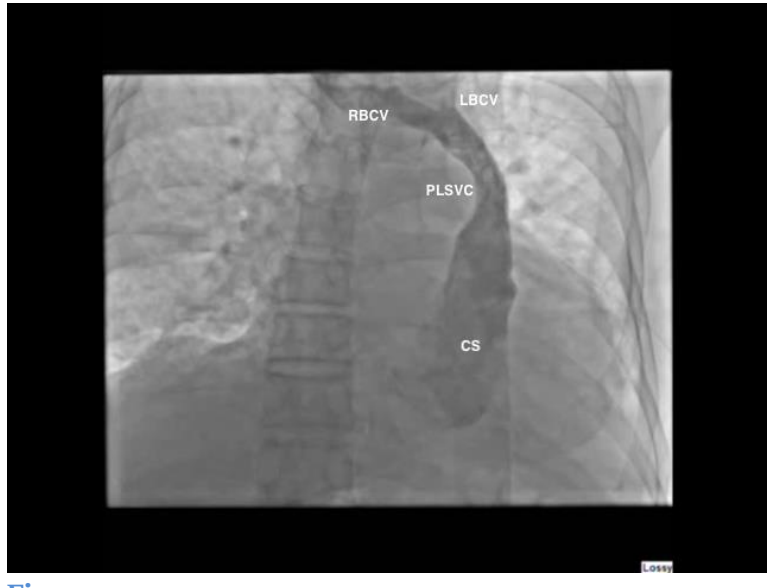

Fig-3:

Venogram with injection from right internal jugular vein confirms persistent left superior vena cava and absent right superior vena cava. The right brachiocephalic vein drains into the left SVC and a very dilated coronary sinus.

RBCV: Right Brachiocephalic Vein; LBCV: Left

Brachiocephalic Vein; PLSVC: Persistent Left Superior Vena

Cava; CS: Coronary Sinus

The video related to this can be found online at: Video-3

\section{Financial Support}

None

\section{Author Contribution}

Diana Otero: Concept and drafting;

Marcus Stoddard: Critical revision and approval or article;

Ikram Sohail: Critical revision and approval or article.

\section{Conflict of Interest}

All authors have read and approved the final version of the manuscript. The authors have no conflicts of interest to declare.

\section{References}

[1] Patel Y, Gupta R. Persistent Left Superior Vena Cava with Absent Right Superior Vena Cava. Methodist Debakey Cardiovasc J. 2018 Jul-Sep;14(3):232-35. [PMID: 30410655] 